\title{
Tracheal size following tracheostomy with cuffed tracheostomy tubes: an experimental study
}

\author{
J. N. LEVERMENT, F. G . P E A R S ON, a nd S. RAE \\ Department of Cardio-Thoracic Surgery, United Bristol Hospitals, England and \\ Toronto General Hospital and Thoracic Surgical Department, University of Toronto, Canada
}

\begin{abstract}
Leverment, J. N., Pearson, F. G., and Rae, S. (1975). Thorax, 30, 271-277. Tracheal size following tracheostomy with cuffed tracheostomy tubes: an experimental study. In view of the severe damage caused by unyielding, low residual volume cuffs, various modifications to the cuff of an intratracheal tube have been introduced. The merits of two lowpressure cuffs were assessed in an experimental study in dogs; both cuffs produced little visible damage to the tracheal wall in dogs intubated continuously over a two-week period.

A modified technique of producing tantalum tracheobronchograms without disturbing the mucous blanket or traumatizing the tracheal wall is described. These tantalum radiological studies demonstrated a progressive temporary increase in size of the trachea at cuff level over the period of intubation with these cuffs. The implications of such a progressive weakness occurring in the tracheal muscle are discussed.
\end{abstract}

Trendelenburg (1871) described the first tracheostomy tube with an inflatable cuff to prevent asphyxia and aspiration during surgical procedures in the mouth and pharynx.

Recently both the seriousness and frequency of occurrence of injury to the trachea by inflatable cuffs in patients requiring tracheostomy and positive pressure ventilation have been stressed (Stiles, 1965; Gibson, 1967; Cooper and Grillo, 1969; Lindholm, 1969; Andrews and Pearson, 1971).

Most publications deal with changes by pressure necrosis on the tracheal mucosa and cartilages; the effect of cuffs on the tracheal musculature needs further investigation.

\section{MATERIALS AND METHODS}

Twenty-seven healthy mongrel dogs $(17-20 \mathrm{~kg})$ with tracheas of comparable sizes were used. Low tracheostomies were performed on 24 of the dogs under general anaesthesia without endotracheal intubation using a single intravenous dose $(20 \mathrm{mg} / \mathrm{kg})$ of a $2 \%$ sodium pentothal solution. The dogs were divided into five groups related to their management:

Group I: Four dogs were intubated with uncuffed tracheostomy tubes.

Group II: Four dogs were intubated with soft, silicone, low residual volume cuffed tracheostomy tubes (experimental design).
Group III: Six dogs were intubated with lowpressure foam cuffed tracheostomy tubes (Kamen and Wilkinson, 1971).

Group IV: Ten dogs were intubated with Foregger, soft, high residual volume, low-pressure cuffed tubes (Grillo et al., 1971).

All the tubes used had internal and external diameters of $7.0 \mathrm{~mm}$ and $9.0 \mathrm{~mm}$ respectively.

Group V: Three dogs without tracheostomies acted as a control group.

Each study extended over a six-week period. Following tracheostomy the animals remained intubated with the particular type of tracheostomy tube for two weeks, after which the tube was removed. The tracheostomy wounds were allowed to heal by primary intention, and the animals were killed four weeks after extubation.

During the two-week period of intubation, the animals breathed spontaneously via their tracheostomies, the cuffs being inflated to withstand an inspiratory pressure of $25 \mathrm{~cm}$ of water without leakage. This tracheal seal was tested by auscultation twice daily after connecting the cuffed tracheostomy tube for a few minutes on a Bennett respirator. (Figure 1 shows the method used to maintain the seal and guard against chronic escape of air from the inflatable cuffs.)

Each animal was caged separately in an airconditioned room. Sovereign humidifiers (model 707 -SM) continuously supplied water vapour mist into each cage. Humidification was monitored by a Honey- 


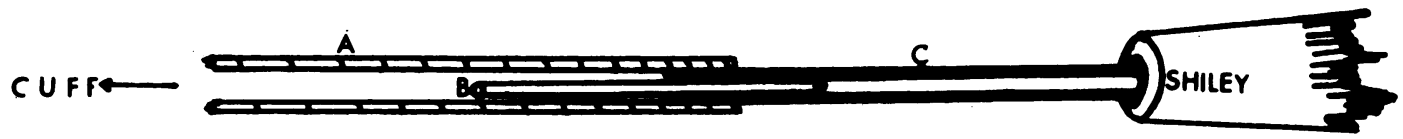

FIG. 1. $2.5 \mathrm{~cm}(I D 0.6 \mathrm{~mm})$ polyethylene tubing $(B)$ interposed between pilot tube to cuff $(A)$ and pilot tube to clamp $(C)$.

well recorder and maintained at $100 \%$ relative humidity at $22^{\circ} \mathrm{C}$. Drinking water was supplied. This method of humidification proved to be adequate because the cannulae remained free of inspissated secretions on each occasion when the tube was removed twice daily for inspection. Faulty cuffs were replaced by new ones. At no stage was endotracheal suction used.

The dogs were bronchoscoped under general anaesthesia prior to tracheostomy and intubation; on the fourth, eight, and thirteenth days of intubation to assess the severity of tracheal injury caused by intubation; and weekly afterwards to observe any residual damage and healing after extubation. The bronchoscopy was done via the larynx using a rigid bronchoscope.

Powdered tantalum, an inert substance, was used to produce tracheobronchograms in order to observe dimensional changes in the tracheobronchial tree. This method of outlining the tracheobronchial tree radiologically was introduced by Nadel, Wolfe, and Graf (1968). A modified technique of using tantulum was used and consisted of a respiratory circuit to ventilate the anaesthetized animal and facilitate deposition of tantalum particles in the lower bronchial tree; and an insufflation circuit to introduce the tantalum without traumatizing the tracheal wall or disturbing the mucous blanket (Fig. 2).

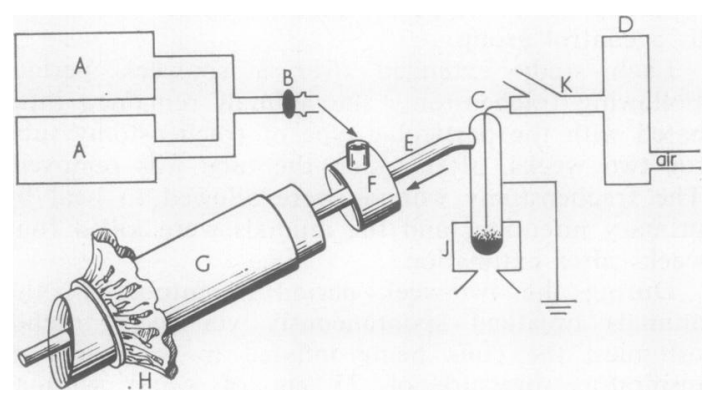

FIG. 2. Apparatus used to deliver tantalum: (A) Harvard respirator; $(B)$ expiratory valve; $(C)$ atomizer containing tantalum; $(D)$ flow meter; $(E)$ polyethylene tubing; $(F)$ swivel connector; $(G)$ endotracheal tube; $(H)$ latex umbrella; $(J)$ vibrator; $(K) \quad Y$-piece connector.

THE RESPIRATORY CIRCUIT A soft Foregger cuff was attached $1.0 \mathrm{~cm}$ above the tip of an uncuffed $9.0 \mathrm{~mm}$ endotracheal tube. The proximal half of this cuff was excised; the cuff then resembled a small umbrella with $\vec{\omega}$ its apex pointing distally. The endotracheal tube was introduced with its tip just past the true vocal cords, $\underset{x}{\times}$ the umbrella being above the cords in order that air could freely escape around the tube during expiration. $\omega$

The endotracheal tube was connected to a Harvard iv respirator and an expiratory flap valve (AMBU) was $\underset{ }{\longrightarrow}$ positioned between the endotracheal tube and respira-음 tor. The valve acted as a warning indicator by its failure to open during expiration when the system $c$ became closed as a result of outflow obstruction, when either the latex umbrella blocked the larynx or the ${ }^{\mathbb{D}}$ tip of the endotracheal tube was displaced too far into $\vec{\theta}$ the larynx.

The animal was respired at a rate of 13.6 respirations per minute. Adequate ventilation was gauged 0 by measuring excursions of the chest wall (Hamlin and Smith, 1967). However, the inspiratory/expiratory ratio was $1: 2$ to ensure complete expiration to prevent a build-up of pressure within the tracheal $\stackrel{\varrho}{\varrho}$ lumen. The same volume setting on the Harvard $\overrightarrow{\vec{A}}$ respirator (average 1,250-1,300 $\mathrm{ml}$ ) was used for each animal throughout the course of its study.

THE INSUFFLATION CIRCUIT From a compressed air source, air entered a flow meter set at 6 litres per minute and then entered an atomizer containing $7 \mathrm{~g}$ of tantalum powder with an average diameter of $5 \times$ microns. The atomizer was grounded to prevent static 3 electrical discharge because tantalum may ignite. under certain conditions. A vibrator agitating the $O$ atomizer ensured an even and rapid insufflation of the tantalum particles. The air containing the tantalum 윽 particles then flowed through a 3.3 metre length of $D$ polyethylene tubing, the proximal end having an inter-을. nal diameter (ID) of $1.67 \mathrm{~mm}$ and the distal end an ID of $3.0 \mathrm{~mm}$, which in turn was tapered to an IDS of $1.67 \mathrm{~mm}$ over the final $1.0 \mathrm{~cm}$ length. This tubing passed through the top of a Portex swivel connector into the lumen of the endotracheal tube and was $\omega$ guided, under fluoroscopy, to pass just beyond the cricoid cartilage and to lie centrally in the trachea. Only by this means was tantalum evenly deposited on the walls of the tracheobronchial tree without? trauma to the tracheal wall or disturbing the mucous 0 blanket. Tantalum was insufflated only during the $\bar{P}$ inspiratory phase of ventilation by thumb occlusion $\mathbb{D}$ of the Y-piece connector situated proximal to the $\mathbb{D}$ atomizer. The latex umbrella prevented deposition of tantalum particles in the pharynx and nasal passages during expiration. During the procedure the trache-尺 
ostome was occluded to prevent excessive escape of inspired air.

The tracheostomy tube was removed 15 to 20 minutes prior to the tantalum insufflation. The procedure took 4 to 5 minutes under general anaesthesia, sodium pentothal $20 \mathrm{mg} / \mathrm{kg}$ intravenously, and fluoroscopic control. Anteroposterior and left lateral radiographs were taken immediately and $\frac{1}{2}$ hour and $2 \frac{1}{2}$ hours later; the dogs were then fully conscious and ambulant. The tracheostomy tube was replaced, the cuff inflated, and the tracheal seal tested prior to returning them into their respective cages. The $x$-ray tube output and anode/film distance remained constant throughout the entire study.

The tantalum studies were performed $(a)$ prior to tracheostomy and intubation; $(b)$ on the third, seventh, and fourteenth days after tracheostomy and intubation; and $(c)$ two weeks and four weeks after extubation. Tantalum and bronchoscopic studies were not performed on the same day on the same animal.

The control animals (group V) had similar studies performed at the same intervals although tracheostomies were not carried out. In addition, each dog acted as its own control by having studies carried out prior to tracheostomy.

\section{RESULTS}

All the dogs remained healthy and none died during the course of the study. The tracheostomy openings were easily identified by fluoroscopy and were well demonstrated on the tracheograms.

Anteroposterior radiographs, taken immediately after the tantalum insufflation, showed progressive ballooning of the trachea during the two-week period of intubation at the site where the cuff had rested. By the end of the period of intubation, further dilatation of the trachea had appeared on either side of the cuff site, that is, in the portion of the trachea not directly in contact with the cuff. The diameters of the tracheas in the control group (group V) and in the dogs intubated with uncuffed tubes (group I) were unchanged.

The degree of dilatation varied with the type of cuff used and also between dogs intubated with the same cuff. When the Kamen-Wilkinson cuff (group III) was used, the degree of dilatation measured on the anteroposterior radiographs was never more than the diameter of the cuff (Fig. 3). Greater degrees of dilatation of the trachea were seen when the Foregger cuff (group IV) was used (Fig. 4). The silicone cuffs (group II) caused less marked dilatations than those seen in groups III and IV; however, these cuffs inflated asymmetrically and bulged mainly towards the anterior tracheal wall (Fig. 5). With each cuff, the extent of dilatation along the axis of the trachea was

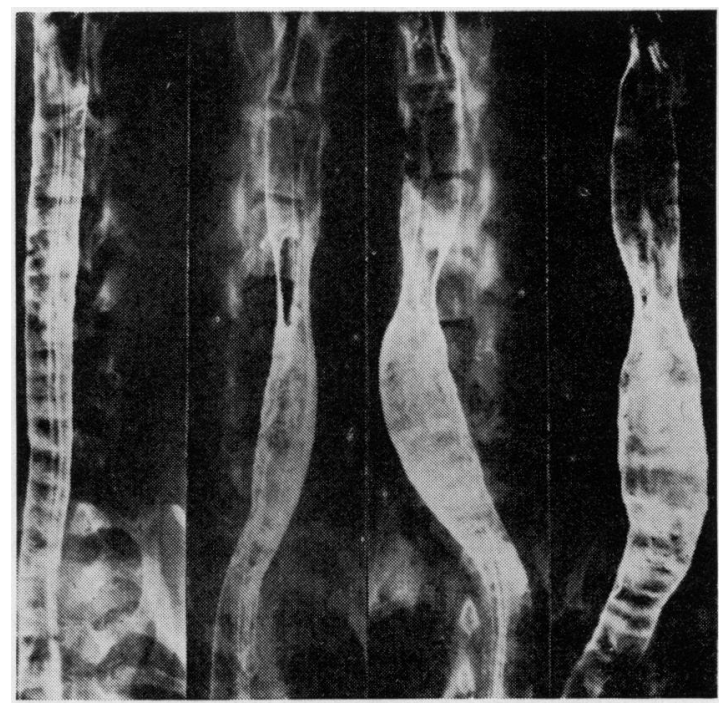

FIG. 3. Tracheal size using Kamen-Wilkinson cuff. Preoperative tantalum study compared with third-day, one-week, and two-weeks' intubation studies demonstrating progressive temporary dilatation (reading from left to right).

invariably longer than the length of the cuff used.

The tantalum particles were gradually cleared from the tracheobronchial tree by mucociliary activity and coughing. However, retention of up to $20 \%$ of the administered dose of tantalum was shown by Upham, Graham, and Steckel (1970). The safety of tantalum in the human is not yet established because of the possible long-term effects of retained tantalum in the lungs and regional lymph nodes. Anteroposterior radiographs taken $\frac{1}{2}$ hour and $2 \frac{1}{2}$ hours after tantalum insufflation demonstrated some residual ballooning of the trachea at cuff level. This weakness of the tracheal wall at cuff level could also be demonstrated two to four days after removal of the tracheostomy tubes by obtaining air tracheograms, without using the tantalum insufflation circuit. Tantalum studies done two weeks after extubation, however, revealed that the trachea had returned to its preoperative size at cuff level.

Bronchoscopic examinations carried out during the period of intubation revealed only superficial mucosal and submucosal damage to the trachea at the cuff site; no cuff site lesions were evident bronchoscopically two weeks after extubation.

At necropsy all dogs showed the expected tracheal narrowing at stomal level. Interestingly, the two dogs with the most impressive dilatation 


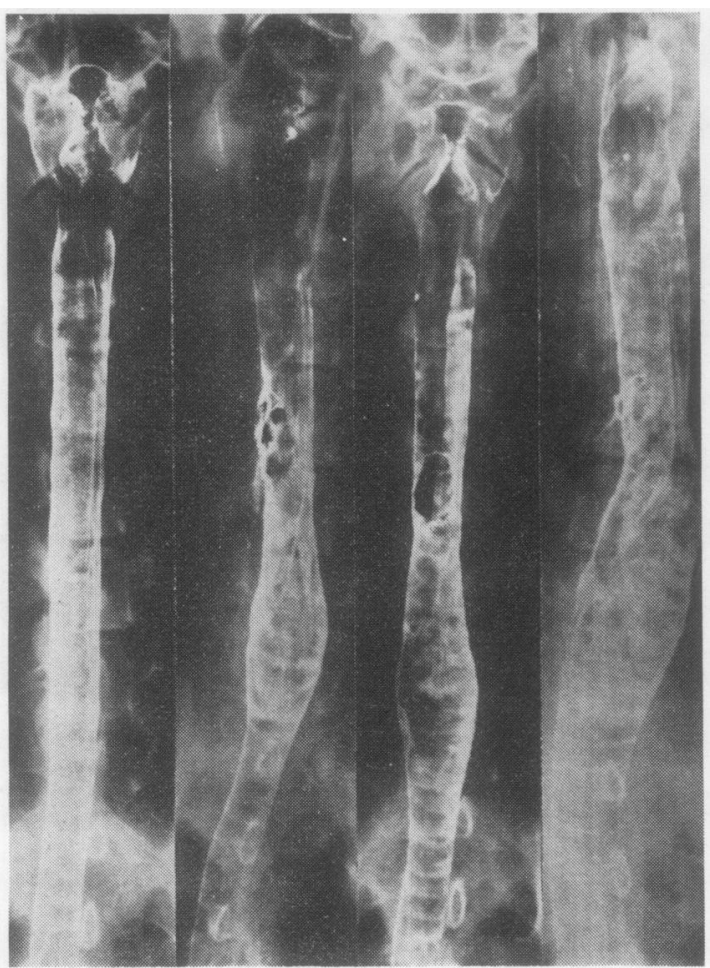

FIG. 4. Tracheal size using Foregger cuff. Preoperative tantalum study compared with third-day, one-week, and two-weeks' intubation studies demonstating progressive temporary dilatation.

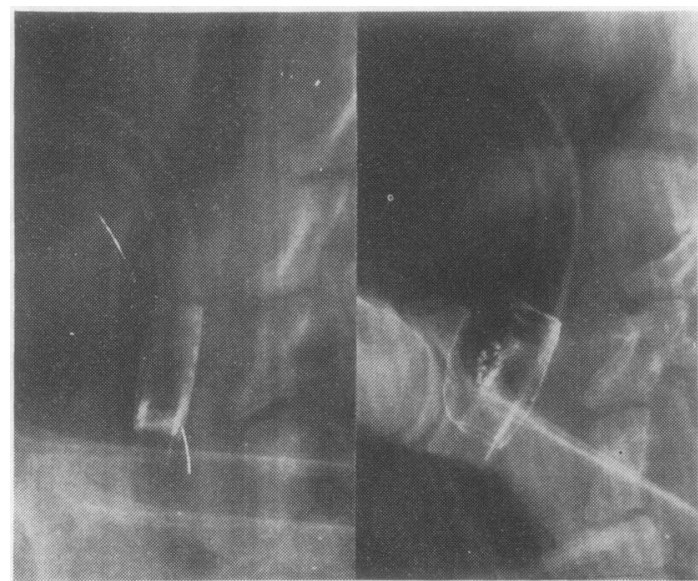

FIG. 5. Soft silicone experimental cuff showing asymmetrical expansion.

of the trachea during their intubation periods demonstrated the worst anterolateral narrowing of the tracheal lumen at stomal level (Fig. 6).

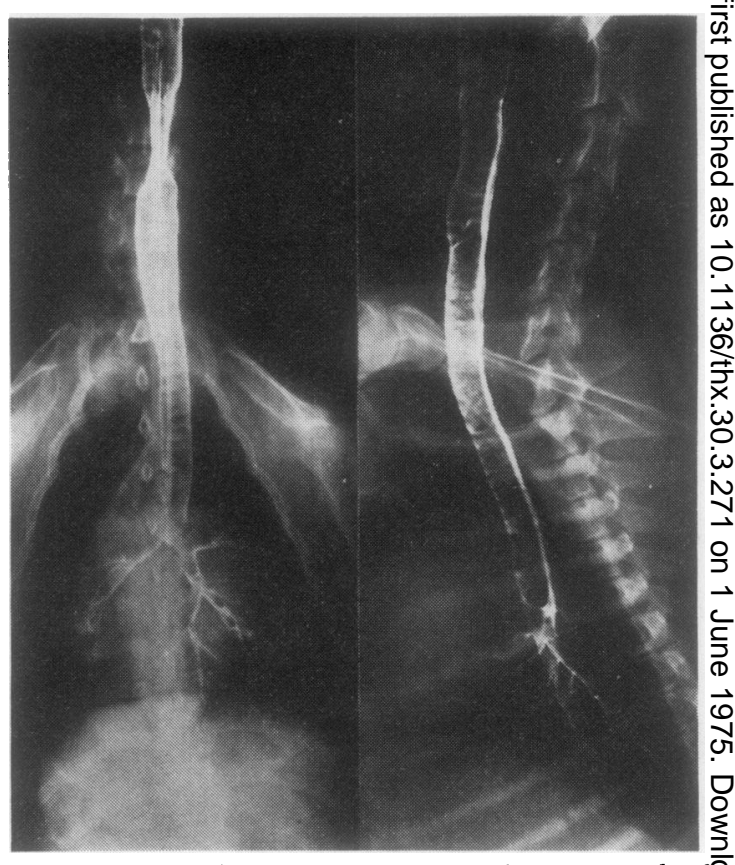

FIG. 6. Anterolateral narrowing of the tracheal lumen at stomal level four weeks after extubation. 응 Adequate lumen on lateral radiograph.

This stomal narrowing was not functionally significant.

\section{DISCUSSION}

It is established that dimensional changes occur in the human and dog trachea in response to dif-o ferent airway pressures (Kilburn, 1960; Croteau. and Cook, 1961). Such uniform dimensionalô changes in volume of the tracheobronchial tree $₹$ were demonstrated in our series of dog experi-음 ments. Localized areas of dilatation or ballooning $>$ of the trachea were also observed below the stoma. These dilatations were still evident $2 \frac{1}{2} N$ hours after cessation of artificial ventilation even though the rest of the tracheobronchial tree had returned to its normal volume.

Conventional cuffs are inflated to provide an airtight seal at the peak of the inspiratory pressureo when the maximum increase in volume has occurred in the tracheobronchial tree. Therefore ${ }^{\text {? }}$ during expiration when decreasing pressure and volume changes occur, a localized area of the $\stackrel{\vec{\odot}}{\circ}$ trachea at cuff site invariably remains 'stretched' by the inflated cuff. Whereas the stiff, unyielding, $\stackrel{\mathbb{Q}}{\stackrel{\Omega}{2}}$ low residual volume cuff produced a tracheal seato by actually deforming the tracheal lumen, the 
newer cuffs (Foregger and Kamen-Wilkinson) supposedly take up the shape of the tracheal lumen without major deformation of the more compliant posterior tracheal wall. The diameter of the trachea at cuff site was only minimally increased on the lateral radiographs (Figures 7 and 8) taken after two weeks of intubation with either the Kamen-Wilkinson or Foregger tube. Tracheal ballooning was more impressively demonstrated by anteroposterior radiographs (Figures 3 and 4), which suggest a weakness in active tension affecting mainly the posterior tracheal wall even when the newer cuffs were used.

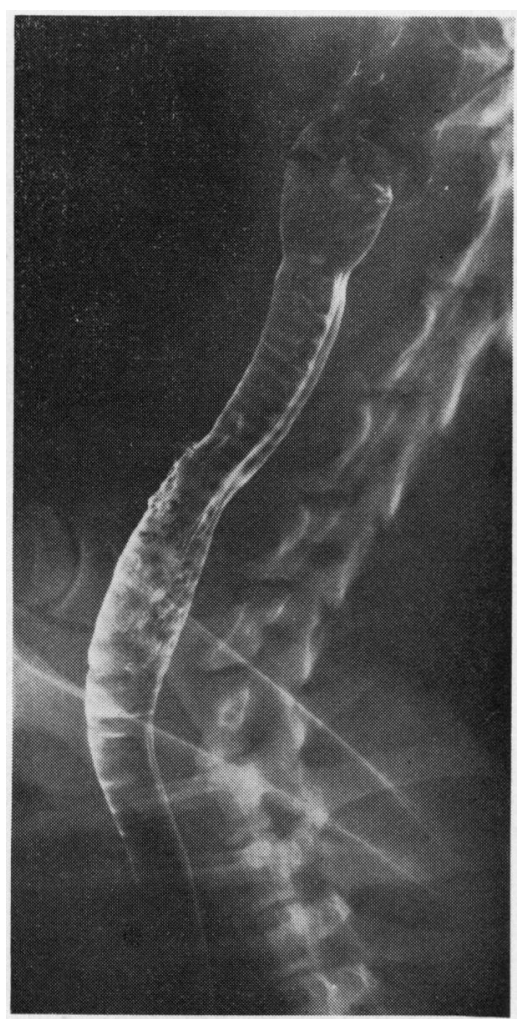

FIG. 7. Lateral tantalum tracheogram (two-week intubation study) showing some dilatation at cuff level (Kamen-Wilkinson).

The Kamen-Wilkinson cuff allows air to escape from the cuff during expiration, minimizing tracheal damage during this phase of respiration. This cuff does not intermittently inflate and deflate when either a leak develops in the sheath covering the foam or the foam itself becomes wet.

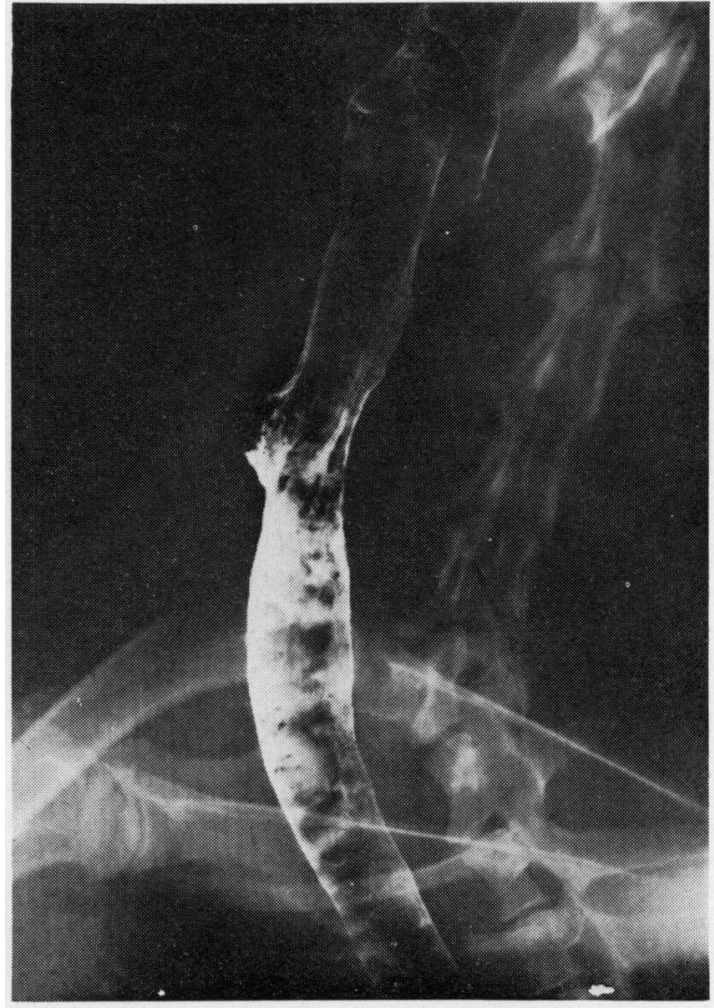

FIG. 8. Lateral tantalum tracheogram (two-week intubation study) showing some dilatation at cuff level (Foregger).

It was hoped that intermittent self-inflating cuffs advocated by Crosby (1964) and Martinez (1964) would cause damage to the tracheal wall only during inspiration, but these cuffs did not safeguard against pulmonary aspiration.

Lomholt (1967) and McGinnis et al. (1971) described cuffed tracheostomy tubes in which the intracuff pressure is maintained at a fixed pressure. The intracuff pressure is mainly related to the characteristics of the cuff used but is also affected by the pressure exerted on it by the tracheal wall. In view of the progressive dilatation of the trachea following prolonged intubation, undesired stretching of the posterior tracheal muscle may result using these devices; especially if the external pressure control system should become faulty, allowing only a one-way flow of air into the cuff.

Widdicombe (1963) presented considerable evidence that the tracheobronchial tree is far from being a rigid or passive duct system. During the expiratory phase of coughing, changes occur in 
the volume of the airways; the importance of reduction in the diameter of the trachea to increase airflow velocity and make the cough effective has been stressed (Ross, Gramiak, and Rahn, 1955).

It has also been suggested that the posterior tracheal muscle of the dog invaginates and squeezes secretions adherent to the tracheal wall into the lumen of the trachea (Coburn, 1972). This effect facilitates clearance of the secretions during a cough and is also dependent on active tension in the tracheal muscle. In the human trachea the effect is controversial.

The dilatation we have observed appears to be due to a temporary change in tracheal muscle tone, because ballooning of the trachea at cuff level could not be demonstrated two to four days after removal of the cuffed tracheostomy tube, and at no stage was severe ulceration or fragmentation of the tracheal cartilages seen bronchoscopically at cuff level. The ineffective cough observed in tracheotomized patients during the weaning period after prolonged positive pressure ventilation may be partly related to this weakness in the posterior tracheal muscle. These patients may be able to clear secretions only if improvement in the tracheal muscle tone occurs. This period may be prolonged in chronic bronchitics in whom the airway muscle is reported to be atrophic.

\section{CONCLUSIONS}

The merits of low pressure cuffs were assessed in this experimental study in dogs. The severity of early tracheal damage at cuff level during the intubation period, and the incidence of late tracheal injury, that is, tracheal stenosis at cuff level after extubation, is far less than when the stiff, unyielding, low residual volume cuffs were used (Goldberg and Pearson, 1972). Even though the Kamen-Wilkinson and the high residual volume Foregger cuffs cause less visible damage to the trachea during intubation, they presumably produced an adverse effect on the posterior tracheal muscle. The Kamen-Wilkinson cuff and intermittent-inflating cuffs attempt to minimize this damage to the tracheal muscle tone. Other cuffs may produce rapid deterioration of the compliance in the tracheal muscle.

Miller and Sethi (1970) introduced an endotracheal tube with flanges replacing the cuff. The merits of this tube as yet have not been established. At present we still rely on an inflatable cuff to provide a tracheal seal during positive pressure ventilation and to prevent aspiration of $\stackrel{\overline{\mathcal{C}}}{\stackrel{\rho}{2}}$ secretions into the lungs. Thus meticulous atrau- 0 matic and aseptic tracheostomy care remains $\underline{\underline{E}}$ important, and the danger of over-inflating any $\frac{\bar{s}}{\vec{D}}$ cuff must be stressed.

\section{REFERENCES}

Andrews, M. J. and Pearson, F. G. (1971). The incidence and pathogenesis of tracheal injury following cuffed tube tracheostomy with assisted ventilation: An analysis of a two-year prospective study. Annals of Surgery, 173, 249. W

Coburn, R. F. (1972). Squeegee of fluid in the com- $Y$ pressed canine trachea. Respiration Physiology, $\vec{\circ}$ 16, 33.

Cooper, J. D. and Grillo, H. C. (1969). The evolution of tracheal injury due to ventilatory assistance through cuffed tubes: A pathologic study. $\overline{\mathbb{D}}$

Annals of Surgery, 169, 334 .
Crosby, W. M. (1964). Automatic intermittent infla- $-\vec{v}$ tion of tracheostomy-tube cuff. Lancet, 2, 509. G

Croteau, J. R. and Cook, C. D. (1961). Volumepressure and length-tension measurements in human tracheal and bronchial segments. Journal of Applied Physiology, 16, 170.

Gibson, P. (1967). Aetiology and repair of tracheal $\stackrel{\circ}{\mathrm{Q}}$ stenosis following tracheostomy and intermittent $\varrho$ positive pressure respiration. Thorax, 22, 1.

Goldberg, M. and Pearson, F. G. (1972). Pathogenesis 3 of tracheal stenosis following tracheostomy with $\underset{ }{ }$ a cuffed tube. An experimental study in dogs. Thorax, 27, 678.

Grillo, H. C., Cooper, J. D., Geffin, B., and Pontoppidon, H. (1971). A low-pressure cuff for tracheostomy tubes to minimize tracheal injury. Journal of Thoracic and Cardiovascular Surgery, 62, 898.

Hamlin, R. L. and Smith, G. R. (1967). Characteristics $ᄋ$ of respiration in healthy dogs anesthetised with sodium pentobarbitol. American Journal of 0 Veterinary Research, 122, 173.

Kamen, J. M. and Wilkinson, C. J. (1971). A new $\frac{D}{2}$ low-pressure cuff for endotracheal tubes. Anesthesiology, 34, 482.

Kilburn, K. H. (1960). Dimensional responses of bronchi in apneic dogs to airway pressure, gases $N$ and drugs. Journal of Applied Physiology, 15, N 299.

Lindholm, C. E. (1969). Prolonged endotracheal intubation. Acta Anaesthesiologica Scandinavica, $\stackrel{\varrho}{\subset}$ Supplement, p. 33.

Lomholt, N. (1967). A new tracheostomy tube. I. Cuff? with controlled pressure on the tracheal mucous membrane. Acta Anaesthesiologica Scandinavica, 11, 311.

Martinez, H. E. (1964). An improved cuffed tracheostomy tube for use with intermittent positive $\frac{\varrho}{\sigma}$ pressure breathing. Journal of Thoracic and Cardiovascular Surgery, 47, 404. 
McGinnis, G. E., Shively, J. G., Patterson, R. L. and Magovern, G. J. (1971). An engineering analysis of intratracheal tube cuffs. Anesthesia and Analgesia, 50, 557.

Miller, D. R. and Sethi, G. (1970). Tracheal stenosis following prolonged cuffed intubation. Cause and prevention. Annals of Surgery, 171, 283.

Nadel, J. A., Wolfe, W. G., and Graf, P. D. (1968). Powdered tantalum as a medium for bronchography in canine and human lungs. Investigative Radiology, 3, 229.

Ross, B. B., Gramiak, R., and Rahn, H. (1955). Physical dynamics of the cough mechanism. Journal of A pplied Physiology, 8, 264.

Stiles, P. J. (1965). Tracheal lesions after tracheostomy. Thorax, $20,517$.
Trendelenburg, F. (1871). Beiträge zu den Operationen an den Luftwegen. Archiv für klinische Chirurgie, 12, 112.

Upham, T., Graham, S., and Steckel, R. J. (1970). Determination of persistence of tantalum dust in vivo following bronchography, using reactoractivated tantalum and total body counting. Investigative Radiology, 5, 289.

Widdicombe, M. S. (1963). Regulation of tracheobronchial smooth muscle. Physiological Reviews, 43, 1.

Requests for reprints to: J. N. Leverment, F.R.C.S., Department of Cardio-Thoracic Surgery, United Bristol Hospitals, Bristol. 